\title{
Petit-déjeuner débat
}

(C) Springer-Verlag France 2012

\section{5}

\section{Traitement chirurgical des hémorroïdes} par la technique HPS, une alternative au doppler

M. Queralto, G. Bonnaud, J.-P. Chotard, J. Levy

Clinique des Cèdres, F-31700 Cornebarrieu, France

Les durées moyennes de séjour doivent se raccourcir, les tutelles imposent pour de nombreux actes une prise en charge ambulatoire par exemple la cholécystectomie. Pour faire face à cette mutation, les techniques chirurgicales deviennent mini-invasives. Bien sûr cette évolution n'est acceptable que si les résultats et la sécurité sont identiques.

La chirurgie proctologique n'échappe pas à cette évolution, la ligature des artères hémorroïdaires avec mucopexie répond à ce nouveau « cahier de charge ».

Classiquement les artères sont détectées par un signal doppler. Le doppler est-il utile?

Une première étude espagnole portant sur 1112 patients montre la faisabilité de l'intervention en ambulatoire [1].

Une étude sur cadavre frais permet de préciser que six artères hémorroïdaires sont localisées à 1, 3, 5, 7, 9 et 11 heures [2].

Une étude simple insu ne retrouve pas de différence significative des résultats à six semaines et six mois pour les interventions réalisées avec ou sans détection doppler [3].

Dans ces conditions, nous avons proposé à nos patients cette nouvelle technique.

Le dispositif HemorPex System [HPS] est un kit se composant d'un anuscope cranté rétroéclairé par une fibre optique insérée dans le manche. Un système de bague crantée permet de venir réaliser une mucopexie par ligature à $1,3,5,7,9$, et 11 heures à l'aide de sutures résorbables fournies dans le kit (aiguille courbe ronde de $22 \mathrm{~mm}$ ).

- La fenêtre crantée permet une mucopexie dans le bon méridien sans chevauchement possible ;

- la fenêtre de petite largeur, l'aiguille $1 / 2$ évite des mucopexies trop larges.

Ce qui permet de réduire la fréquence des faux besoins, des complications à type de fissure ischémique.

Après près de deux ans d'utilisation, nous retenons comme indications :

- les rectorragies imposant des transfusions chez des patients sous AAP/AVK chez lesquels l'interruption n'est pas souhaitable, quel que soit le type des lésions ;

- les prolapsus hémorroïdaires dont le volume ne gêne pas la réalisation de l'intervention (grades 2 et 3, grade 4 de faible volume).

Le traitement des hémorroïdes avec la technique HPS est simple, sûr, efficace, peu douloureux permettant d'être réalisée en ambulatoire, la courbe d'apprentissage est rapide.

\section{Références}

1. Iachino C, Guerrero Y, Sias F, et al (2009) Hemorpex system: a new procedure for the treatment of haemorrhoids. Cir Esp 86:105-9

2. Schuurman JP, Go PM, Bleys RL(2009) Anatomical branches of the superior rectal artery in the distal rectum. Colorectal Dis 11:967-71

3. JP Schuurman, HMIH Borel Rinkes Inne H (2012) Hemorrhoidal artery ligation procedure with or without Doppler transducer in grade II and III hemorrhoidal disease: a blinded randomized clinical trial. Ann Surg 255:840-5 